\title{
Learning Nutrition from Nutrient Sciences of History
}

\author{
Ying Gao-Balch* \\ Department of Human Sciences, The University of Arkansas at Pine Bluff, 1200 North University Drive Pine Bluff, Arkansas, USA \\ *Corresponding author: Ying Gao-Balch, Department of Human Sciences, The University of Arkansas at Pine Bluff, \\ 1200 North University Drive Pine Bluff, Arkansas, USA, E-mail: gaobalchy@uapb.edu
}

\section{DISCOVERY OF VITAMIN B1}

Modern nutritional science is surprisingly young. Nevertheless, there are many nutrition sciences be participating in the nutrition science of the development of nutrition science history. Less than 100 years ago, Nutrition research started observing human clinical or animal clinics. Otherwise, nutrition sciences history has been beginning from lab experimenting.

McCollum and Davis started to observe that the lab rats eat some food to lead to poor growth, so they want to prove the reason for failure in development. Experimenting with two different rats' diets, added to only butterfat and an additional extra of the diet supplement. Result added to the addition of the supplement of diet is to heal and expect growth (Mcllum, 1917). In another story, Chemist Casimir Funk noticed the symptoms of beriberi in chickens. Then he used chickens in the laboratory to feed leftover rice with processed until a new cook changed brown rice. The chickens soon recovered from beriberi. The birds got well again a few days after switching to brown rice to birds. Eijkman thought that processing rice lacked a dietary component found in brown rice. The beriberi caused by an absence in the dietary ingredient, which he called "the anti-beriberi factor." Korsakoff studied the effects of alcoholism on the nervous system and drew attention to several cases of alcoholic polyneuropathy with distinctive mental symptoms (Korsakoff's syndrome) due to the defense of Thiamin. Finally, Eijkman proved that the disease was not caused by blood contamination, respiratory metabolism, or temperature variation. Eventually, it was determined that the missing compound causing beriberi was vitamin B1, whose name is Thiamine. Eijkman with Sir Frederick Hopkins won the 1929 Nobel Prize for Medicine; interest in extracted food nutrients began in the latter half of the 19th century with Thiamin's first vitamin. Thiamin was water-soluble.

\section{VITAMIN B2}

Following Thiamine of the discovery, researchers realized that one or more additional water-soluble factors called the vitamin B2 complex. Observing a pigment in milk with yellow-green, whole wheat, yeast, and liver began by chemist Alexander Wynter Blyth in 1872. Until late 1991, Riboflavin was the second vitamin to be isolated. They thought no nutritional disease causes riboflavin deficiency until they experimented with young Rats to feed with the food extract and observed that the young growth is outstanding to prove Riboflavin's growth-stimulating properties. Riboflavin is the precursor of the coenzymes Flavin adenine dinucleotide (FAD) and flavin mononucleotide (FMN). Several oxidation-reduction (redox) reactions are involved in energy production, and numerous metabolic pathways act as electron carriers. Riboflavin deficiency can affect multiple paths in the metabolism of vitamin B6, folate, niacin, and iron. Riboflavin deficiency has been linked to preeclampsia in pregnant women. This condition may progress to eclampsia and cause severe bleeding and death. The risk of preeclampsia has recently been associated with the presence of a genetic variant (C677T) in the methylenetetrahydrofolate reductase (MTHFR) gene. This gene codes for the MTHFR enzyme, which is FAD-dependent.

Riboflavin deficiency is extremely rare in the United States. Riboflavin deficiency: The signs and symptoms include skin disorders, hyperemia and edema of the mouth and throat, angular stomatitis, cheilosis, hair loss, reproductive problems, sore throat, itchy and red eyes, and degeneration of the liver and nervous system. People with riboflavin deficiency typically have deficiencies of other nutrients, so some of these signs and symptoms might reflect these other deficiencies. Severe riboflavin deficiency can impair the metabolism of other nutrients, especially other B vitamins, through diminished levels of flavin coenzymes. Anemia and cataracts can develop if riboflavin deficiency is severe and prolonged. 


\section{VITAMIN B6}

In the 1930s, Rudolf Peters showed that young rats kept on a semi-synthetic diet with added Thiamin and Riboflavin and no other supplement, so rats developed 'rat acrodynia,' a condition characterized by severe cutaneous lesions. Then, in 1934, the Hungarian physician Paul György discovered a substance that could cure a skin disease in rats (dermatitis acrodynia). He named this substance vitamin B6. In 1938, Samuel Lepkovsky isolated vitamin B6 from rice bran. In 1934, Paul György showed that the factor which cured 'rat acrodynia' was vitamin B6. However, other studies soon showed that vitamin B 6 deficiency produced convulsions in rats, pigs, and dogs and microcytic anemia in certain animals. Samuel Lepkovsky isolated and crystallized vitamin B6 in 1938. The following year, Leslie Harris and Karl Folkers, Richard Kuhn, and his associates independently showed that vitamin B6 was a pyridine derivative 3-hydroxy-4, 5-dihydroxymethyl-2-methyl-pyridine. The pyridoxal, pyridoxamine, and pyridoxine convert into the enzymatically active form of pyridoxal-5-phosphate that plays a role in various enzyme systems, especially in amino acids metabolic utilization and transformation.

\section{VITAMIN B12}

During the 1920s, George Whipple discovered that feeding large amounts of raw liver to cure the blood loss anemia in dogs most rapidly. They also identified the hypothesis that eating the liver might treat pernicious anemia. Edwin Coh prepared a liver extract that was 50 to 100 times more potent in treating pernicious anemia than natural liver products. William Castle discovered that gastric juice contained an "intrinsic factor," a glycoprotein that formed a complex with vitamin $\mathrm{B}_{12}$, promoting its absorption through ileal receptors. Vitamin B12 works with many other B vitamins to carry out critical roles in the functions of the human body. For example, vitamin12 combined with folate and vitamin B6, cobalamin helps maintain normal blood homocysteine levels. The valuable contribution of clinical reports and studies of patients with pernicious anemia throughout the 19th century resulted in enough clinical definition to allow the treatment of the condition, leading them to a Nobel Prize. Minot and Murphy1964.

Subsequently, the various biochemical roles of vitamin $\mathrm{B}_{12}$ were elucidated, including its essential interaction with folate and their common link with megaloblastic anemia. In addition, many early clinical studies recognized that vitamin $\mathrm{B}_{12}$ deficiency also caused severe neuropathy leading to paralysis and death. At the same time, post mortem analysis demonstrated spinal cord demyelination. However, vitamin $B_{12}$ is still the subject of intense research, and, in particular, its role in preventing these irreversible neurological lesions remains unclear.

\section{FOLATE}

In the 1920s, scientists recognized that folate deficiency and anemia were the same conditions. Lucy Wills' research on anemia in pregnant women in 1928 led to folic acid that helps prevent congenital disabilities in babies. She conducted seminal work in India in the late 1920s and early 1930s on macrocytic anemia during pregnancy. During the research on pregnant textile workers in Mumbai, her observations led to discovering a nutritional factor has both prevents and cures this disorder. In 1931, researcher Lucy Wills made a critical observation that identified folate as the nutrient required to avoid anemia during pregnancy. In addition, wills demonstrated that anemia could reverse anemia with brewer's yeast. In the late 1930s, folate was discovered as the substance in brewer's yeast. It was first isolated by extraction from dark-green leafy vegetables. The extract, later identified as folic acid, improved the monkeys' health during the research, named the Wills Factor.

Macrocytic anemia is characterized by enlarged red blood cells and is a life-threatening condition. In 1960, researchers linked folate deficiency to the risk of neural tube defects. In the early 1990s, well-designed randomized trials established that folate supplementation could prevent neural tube defects. Therefore, 62 countries have started to be mandate food fortification with folic acid in December 2018.

\section{VITAMINE C}

Centuries ago, sailors experienced such swollen and bleeding gums, loose teeth, hemorrhaging under the skin, and slowed healing of wounds for extended distances without fresh food and supplies. This medical condition called scurvy was cited as the primary cause of disability and mortality among sailors on long sea voyages. By 1753 scurvy was recognized in the British medical community as directly related to dietary deficiency. In 1769, William Stark, a young British physician, used himself as the experimental subject on diet and nutrition; he ate only bread and water for 31 days. After adding other foods to his diet with olive oil figs, goose meat, and milk. His clinical examed recorded that his gums were red and swollen, bleeding easily to touch for 60 days. Seven months later, he self-sacrificed his life for this experiment. His diet was the highest fat of meat and starch without citrus fruits and fresh vegetables.

It took more years, Albert Szent-Györgyi isolation of vitamin $\mathrm{C}$, an essential substance for use within cells for the metabolic system. Nevertheless, he is recognized with a Nobel Prize in physiology medicine. 


\section{VITAMIN A DISCOVERED}

Many researchers were studying the nutritional requirements of mammals in the early 1900s. Experiments were carried out on young rats and mice' diets, beginning with simple dietary mixtures and finding out what should be more needed. Researchers found out that failures growth resulted from the absence of ingredients in the diet for a long time.

Gowland Hopkins fed young rats on casein, lard, sucrose, starch, and minerals as well half of the rats were also fed by milk daily. Those fed by the milk grew well better than no fed by the group's milk after two weeks; he found that those receiving the milk usually grow, and those lacking did not develop well. He explained this by the basic diet lacking some essential organic nutrients. He felt that similar problems might be present in human diseases related to diet.

Elmer McCollum found that rats started to heal given a purified diet and started to lose weight after ten weeks but would recover after feeding allotted butter fat, but not olive oil. In 1914 he found out that the active component could be separated from the rest of the butterfat, as it was fat-soluble in ether. He called this nutrient "factor A." These fat-soluble factors later became vitamins A. Vitamin deficiency is related to humans, and rats cause malnutrition, and Vitamin A deficiency can result in severe eye damage. Night blindness, a condition is a person cannot see in dim light, has been known since ancient Egyptian times. However, this has recently been disputed. Certainly, Hippocrates, who lived 460-325 BC, recommended eating raw liver to cure night blindness with nutrients, thus identifying a link between night blindness and nutrition. In addition, many physicians and scientists discovered night blindness, often with malnutrition.

People's nutrient deference is related to dryness of the cornea and conjunctiva (xerophthalmia). Osborne and Mendel's experimental work discovered one essential nutritional factor in these foods, and McCollum and Davis named it "extra fatsoluble factor," later called "fat-soluble vitamin A. Therefore, preventive interventions such as dietary nutrients with vitamin A enrichment.

They successfully restored about thirty rats to normality after reaching the growth suspension stage by adding a small number of extracts of egg or butter. Their paper included charts for five rats, illustrating their weights over time compared to an average growth curve; one chart showed their results with a fat-free diet; rats could not grow without a substance in the egg or butter extract, even though they appeared to be healthy. They concluded that rats stop growing until they fed certain "ether extracts of egg or butter" of essential for average growth for extended periods." The first is that a lack of nutrients in the diet components could be harmful. Second, Gowland Hopkins had isolated and discovered the essential nature of the amino acid tryptophan molecule. They also found this food factor in extracts of alfalfa leaves and organ meats. McCollum called "factor A" was later called vitamin A.

\section{VITAMIN D}

A Johns Hopkins pediatrician thought that rickets was caused by diet. McCollum's research during the early 1920s found that rats fed with a plain diet led to developing rickets. After that, His group tested more than 300 diets on rats, finally finding that cod-liver oil could prevent rickets. Further, more tests found that the oil could no longer cure night blindness but can cure rickets in rats. After that, he named the substance for the following free letter of the alphabet, vitamin D. Furthermore, they tested that sunshine and cod liver oil protected against rickets and tested this by carrying children outside to the sun. Soon after, many children grew up with fed cod-liver oil; these children of rickets completely went away.

The discovery in 1919-1924 of vitamin D and its production in skin and foods by UV irradiation to eliminate rickets is a significant medical problem. The physician used vitamin D to mineralize the skeleton to prevent rickets in children and osteomalacia in adults early in the 1960s. For applying biochemistry to discover that vitamin D must first be activated by 25 -hydroxylation in the liver followed by one alphahydroxylation in the kidney to produce the vitamin $\mathrm{D}$ hormone one alpha,25-dihydroxy vitamin D3 [1,25-(OH)2D3]. This process is strongly feedback-regulates plasma calcium and phosphorus concentrations. Furthermore, it is a primary endocrine system regulating bone mass and state. Using 1,25-(OH)2D3, Understanding how this hormone facilitates calcium transport across the intestinal membrane keeps in mineral homeostasis.

\section{VITAMIN E}

In 1920 Mattel and Conklin investigated milk as the "perfect food." They wanted to determine "if milk could be an adequate food for the whole span of life for the normal performance of a physiological function." They fed a diet of fresh whole milk to rats for their entire life span. The rats grew well initially; after 50ds, growth declined, and the females did not reproduce. Given whole dried milk, obtained commercially, food consumption was more significant than fresh milk. Again, growth was average, but there was no reproduction. When $10 \%$ butterfat was added to the diet, replacing that amount of starch, the rats were still sterile.

Vitamin E in infant nutrition has a role in long research history. They used oral alpha-tocopherol feed with premature infants. 


\section{Short Commentary}

The result of vitamin $\mathrm{E}$ supplementation reduced the risk of intracranial hemorrhage and retinopathy. However, it noted an increased risk of sepsis in preterm infants. However, no proven to women miscarriage.

Vitamin E ( $\alpha$-tocopherol) was found nearly 100 years ago. The human diet has eight different vitamin E-related molecules synthesized by plants; despite these molecules being per-oxyl radicals scavengers, the human body prefers $\alpha$-tocopherol. The biological activity of vitamin E depends on regulatory mechanisms that retain $\alpha$-tocopherol and excrete the non- $\alpha$-tocopherol. In addition, $\alpha$-Tocopherol functions as a radical scavenger to protect polyunsaturated fatty acids and lipoproteins. The most likely explanation for why humans require vitamin $\mathrm{E}$ is a fat-soluble antioxidant. Vitamin $\mathrm{E}$ was first isolated in a pure form by Evans and Gladys Anderson Emerson in 1935 at the University of California, Berkeley.

Citation: Gao-Balch Y (2022) Learning Nutrition from Nutrient Sciences of History. Appl Cell Biol, 10(1), 2022 [18-21] 